\title{
Calculation of Bag's Constant Using Planck's Equation of Black Body Radiation
}

\author{
Arijit Bag \\ Department of Chemical Sciences, Indian Institute of Science Education and Research Kolkata, Mohanpur-741246, West Bengal, India
}

\begin{abstract}
Classical interpretation and derivation of wave particle duality relation has been done very recently using Newtonian mechanics which is termed as Bag's wave of a particle. According to Bag's wave particle duality, wavelength of a moving particle is $\lambda=b$ $/ p$, instead of $\lambda=h / p$ which is for de Broglie wave length of a particle. The constant ' $b$ ' is a function of opposition field and spin momentum of the particle. So far $b$ is not characterized completely and not used for experimental purpose. In this article an approximate value of $b$ is presented which is calculated using few assumptions. Calculated value of b differs from value of h. This discrepancy is addressed. It is also shown that at $T=4.8 \times 10^{-11} \mathrm{~K}$, $\mathrm{b}$ and $\mathrm{h}$ become equal. This temperature is termed as equivalence temperature.
\end{abstract}

Keywords: Bag's constant; Black body radiation; Wave particle duality; Equivalence temperature.

\section{Introduction}

In my very recent work [1], wave nature of a moving particle is derived using Newtonian mechanics. Derived relation between momentum and wave length of a particle using classical mechanics is similar to de Broglie's wave particle duality relation [2]. In both the cases wave length of a particle is inversely proportional to the momentum of that particle. But, proportionality constants of these two methods may not be same. So far, no study is done for comparison. In de Broglie's wave particle duality relation Planck's constant $h$ is used, but in Bag's method $b$ is used as proportionality constant. By nature, $b$ is a constant only when applied field and spin momentum of the particle is fixed while $h$ and de Broglie's wave length of a particle is independent of field. Thus, we can consider that $h$ is only a specific value of $b$ at a particular condition. In this article, classical form of wave particle duality relation is incorporated into Planck's equation of black body radiation, to find an expression to calculate $b$.

\section{Theory}

According to the Planck's postulates [3] about the quantization of black body radiation, a black body is a collection of several resonators. It absorbs or emits energy in a quantized way. Frequency of absorbed or emitted energy is equal to the vibrational frequency of a resonator. Thus, emission of a black body is due to the vibration of the resonators. A particular resonator has two degrees of freedom. Again, from equi-partition principal we know that kinetic energy of one mode of vibration at a temperature $T$ is (1/2) $k T$, where $k$ is Boltzmann constant. On the other hand kinetic energy of a particle having mass $m$ and momentum $p$ is $p^{2} / 2 m$. Thus, equating these two conditions we get the following relation for a resonator

or,

$$
\text { (1/2) } k T=p^{2} / 2 m
$$

$$
k T=p^{2} / m
$$

Now, according to classical wave particle duality relation i.e. for Bag's wavelength $(\lambda)$

$$
p=b / \lambda
$$

where $\lambda$ is wave length of the resonator. Equation 3 may be written in terms of frequency $v$ as

$$
p=b v / c
$$

where $c$ is the velocity of light in vacuum. Putting the value of $p$ from equation 4 in equation 2 we get

$$
k T=\left(b^{2} v^{2}\right) /\left(m c^{2}\right)
$$

In equation $5, m$ is mass of the resonator and $c$ is the velocity of light. Hence according to Einstein's energy mass equivalence relation [4],

$$
m c^{2}=E
$$

and considering the energy quantization we can write

$$
E=h v_{0}
$$

Here $v_{0}$ is different from $v . v_{0}$ is in fact mass of the resonator written in terms of frequency. Thus, from equation 5, equation 6 and equation 7 we can write

$$
b=\sqrt{ }\left(h v_{0} k T / v^{2}\right)
$$

Equation 8 is the relation we want to evaluate Bag's constant $b$. But, in equation $8, v_{0}$ is difficult to evaluate. If we get any relation between $v$ and $v_{0}$ from any other method or experimental results, calculation of $b$ would be possible. In equation $8, h$ and $k$ are constants; $v_{0}$ is not defined i.e. whether it is a fixed quantity or not. It is possible that in a black body, resonators of different masses are present but mass of a particular resonator does not change with change of temperature. In that case we may consider $v_{0}$ as constant if we focus our study on a chromatic radiation.

\section{Numerical Analysis}

To calculate $b$, we may consider different limiting conditions. First we may calculate $b$ for $v=1 \mathrm{~Hz}, v_{0}=1 \mathrm{~Hz}$ and $T=1 \mathrm{~K}$. Using $h=6.62607 \times 10^{-34} \mathrm{JS}$ and $k=1.3806 \times$ $10^{-23} \mathrm{JK}^{-1}$ we get $b=9.56 \times 10^{-29} \mathrm{JS}$ which is higher than the value of Planck's constant $h$ by a factor nearly $10^{5}$. This 


\section{International Journal of Science and Research (IJSR) \\ ISSN (Online): 2319-7064}

Index Copernicus Value (2013): 6.14 | Impact Factor (2014): 5.611

discrepancy may be due to the wrong choice of limiting conditions. It is well known that with increase of temperature frequency of emission increases. Thus, there is a possibility that $b$ may be a constant with increase of temperature if increase of emission frequency follow the relation

$$
v^{2}=\sqrt{ } T
$$

Else, change of $b$ with change of temperature would depend on the relation between $v$ and $T$.

We may calculate the value of $\mathrm{T}$ at which $b=h$. Let $T_{e}$ be the equivalence temperature. Then from equation 8 we get

$$
b=k T_{e} v_{0} / v^{2}
$$

Now, at very low temperature i.e. when $T \rightarrow 0$, all resonators would be at their ground state energy level. Let $x$ is the value of lowest possible frequency of any electro magnetic radiation. Then, if a resonator of lowest possible mass exists in its lowest energy state, we should get both $v=x$ and $v_{0}=$ $x$. For finite mass, $\mathrm{x}=1 \mathrm{~Hz}$. Thus, from equation 10 we get,

$$
b=k T_{e}
$$

we have consider that at $\mathrm{T}_{\mathrm{e}}, b=h$. hence,

$$
T_{e}=h / k
$$

Putting the value of $h$ and $k$ in equation 12, we get the equivalence temperature $T_{e}$ as $T_{e}=4.8 \times 10^{-11} K$. Thus we can conclude that only at very low temperature region Bag's constant and Planck's constant would be equivalent.

\section{Conclusions}

In this article, nature of proportionality constant (b, Bag's constant) of classical weave particle duality relation is discussed. Though estimation of exact value of $b$ is not done here, a mathematical formulation is given from which one can calculate $b$ experimentally. But, more importantly, it is presented that at a temperature $4.8 \times 10^{-11} \mathrm{~K}, b$ and $h$ becomes equal. $b$ may not vary with temperature if increase of emission frequency with increase of temperature follows equation 9. The complete description of $b$ would be possible if we be able to do experiment to get unknown variables of equation 8 . At present we can only conclude that by nature, $b$ is same as $h$ but numerical value of $b$ may be different and will depend on some external parameter like field strength, and internal parameter like spin momentum of a system. Following equation $8, b$ would be an universal constant only if

$$
v^{2}=\sqrt{ }\left(v_{0} T\right)
$$

for all temperature.

\section{Acknowledgments}

I acknowledge IISER Kolkata, India, for funding and research facilities and Dr. Pradip Kr. Ghorai, Associate Professor, IISER Kolkata to allow me to publish this work independently.

\section{References}

[1] Bag, 2015. Int. J. Res. Sc. Technol. 5 (IV) : 68-72. webwww.ijrst.com

[2] de Broglie, L. 1925. Ann. De Physique 3: 22

[3] Planck, M. 1901. Annalen der Physik, 4, 553

[4] Einstein, A. Sitzungsber Preuss Akademia Wiss, 142 (1917)

\section{Author Profile}

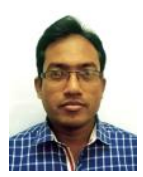

Dr. Arijit Bag get his Master's Degree in Chemistry from University of Calcutta, West Bengal, India in 2001. He joined as a lecturer at Sundarban Mahavidyalaya in 2001 and continued his teaching till December 2003. In 2004, he joined Dr. Sourav Pal's group of theoretical chemistry at National Chemical Laboratory, Pune, India. He did his $\mathrm{Ph}$. D. in Linear response approach to calculate molecular properties using Fock-space Multi-reference Coupled Cluster Singles and Doubles (FSMRCCSD). At present he is doing his post doctorate research at Indian Institute of Science Education and Research Kolkata, West Bengal, India. So far, he had published 12 research papers in several international journals including ELSEVIER Journal of Catalysis, AIP Journal of Chemical Physics, RSC Advances etc. He is a multidimensional researcher having reputed publications in Astrophysics, Quantum mechanics, Molecular and Theoretical Biology, Sociology and Humanities, Molecular Simulation and Drug Designing, Catalysis and Medical methodology. Beside scientific works, he had published three books of poem and two books of drama (in Bengali). He edited a Bengali science magazine for school children. He had also published a music album in which he himself acts as a lyricist, musician and vocalist. He was honored as winner of Bijan Bhattacharya Smriti Puraskar given by All Indian Children Association in 2012 for his collection of short dramas Haste Haste Bhabi. 